\title{
Ultrasound-Guided Transoral Videolaryngoscopic Surgery for Retropharyngeal Lymph Node Metastasis of Papillary Thyroid Cancer
}

\author{
Kazunori Fujiwara Takahiro Fukuhara Satoshi Koyama Ryohei Donishi \\ Hideyuki Kataoka Hiroya Kitano Hiromi Takeuchi \\ Department of Otolaryngology, Head and Neck Surgery, Faculty of Medicine, \\ Tottori University, Yonago, Japan
}

\section{Keywords}

Ultrasound · Transoral surgery · Retropharyngeal lymph node $\cdot$ Papillary thyroid cancer

\begin{abstract}
Background: Endoscopic-assisted transoral surgery, including transoral robotic surgery for metastatic retropharyngeal lymph node (RPN) from well-differentiated thyroid cancer, has been reported to reduce the complications resulting from transcervical and transmandibular approaches. However, the narrow working space and difficulty identifying RPN are problematic. To solve these issues, several studies have used intraoperative ultrasound in endoscopicassisted transoral surgery. However, the type of ultrasonography suitable for this purpose remains unclear. Case Presentation: A 60-year-old female with thyroid papillary carcinoma (T4aN1bM0) initially underwent total thyroidectomy and paratracheal and selective neck dissections (D2a), with resectional management of recurrent laryngeal nerve, trachea, and esophagus. Three years later, she was diagnosed with left retropharyngeal and upper mediastinal lymph node metastases of papillary thyroid cancer. Transoral videolaryngoscopic surgery was performed with a combination of ultrasonography with a flexible laparoscopic transducer manipulated with forceps for identifying RPN intraoperatively. Due to the transducer's small size and thin, flexible cable, the transducer interrupted the procedure in spite of the narrowness of oral cavity. RPN was resected completely without adverse events. Conclu-
\end{abstract}


sion: We performed intraoperative ultrasound-guided endoscopic transoral surgery for metastatic RPN from papillary thyroid cancer and achieved complete resection as well as preservation of swallowing function.

\section{Introduction}

Papillary thyroid cancer (PTC) often shows lymphatic spread, and the incidence of nodal metastasis in differentiated thyroid cancer ranges between 40 and $75 \%$ [1]. Nodal involvement commonly occurs in the central compartment and lateral regions of the neck; however, metastases to the retropharyngeal lymph node (RPN) are rare. Few reports have described RPN metastasis from PTC [2].

Traditionally, surgical excision of isolated RPN metastasis from well-differentiated thyroid carcinoma has been well described via transcervical and transmandibular approaches [3]. These lengthy surgeries, which access the parapharyngeal space, subject patients to increased risks with potentially serious complications, especially facial nerve paralysis, malocclusion, and first bite syndrome. These procedures carry rather high morbidity rates considering the relatively indolent nature of well-differentiated thyroid cancer. Therefore, endoscopic-assisted transoral resection, including transoral robotic surgery, for metastatic RPN from well-differentiated thyroid cancer has recently been described [4-15]. However, the narrow working space and difficulty identifying RPN are problematic. To solve these problems, we introduce here the use of ultrasound during transoral resection of RPN in PTC. Intraoperative ultrasound has been used in conjunction with a finger grip probe and endocavitary transducer. However, the use of a flexible laparoscopic transducer manipulated with forceps has not been reported in transoral surgery. The type of ultrasonography suitable for endoscopic-assisted transoral surgery remains unclear. The current report presents a patient with recurrent metastatic RPN who underwent transoral dissection using intraoperative ultrasound with a flexible laparoscopic transducer manipulated with forceps.

\section{Case Report}

A 57-year-old female was diagnosed at an outside institution 3 years previously with PTC with invasion into the recurrent laryngeal nerve, strap muscle, esophagus, and trachea, as well as multiple lymphadenopathy (T4aN1bM0). The patient was obese (body mass index: 35.0) and had diabetes. Her performance status was 0 according to the Eastern Cooperative Oncology Group system, and she did not smoke or drink.

The patient was referred to our department for treatment. She underwent total thyroidectomy and paratracheal and selective neck dissections (D2a), with resectional management of the recurrent laryngeal nerve, trachea, and esophagus. She also underwent concurrent reconstruction of the recurrent laryngeal nerve. Three months later, her tracheostomy stoma was closed with a local flap, and radioiodine treatment was performed. After that, she received follow-up care at another institution. Three years after her initial treatment of PTC, fluorodeoxyglucose positron emission tomography demonstrated multiple foci of increased fluorodeoxyglucose uptake in the left RPN and upper mediastinal lymph nodes (Fig. 1). The patient was referred to our department for treatment of lymph node metastases. She complained of no symptoms associated with the retropharyngeal or upper mediastinal lymph nodes. Contrast-enhanced T1-weighted magnetic resonance imaging of the neck showed 
lymph node enlargement $(18 \times 14 \mathrm{~mm})$ in the poststyloid component of the parapharyngeal space without invasion of the carotid artery, as well as in the upper mediastinum up to the innominate artery without invasion of the adjacent tissue (Fig. 2). Cytology of the upper mediastinal lymph nodes revealed papillary adenocarcinoma. No cranial nerve deficits were observed. The patient was diagnosed with left retropharyngeal and upper mediastinal lymph node metastases from PTC. She underwent upper mediastinal dissection and left retropharyngeal dissection concurrently. The upper mediastinal nodes were resected successfully through the cervical approach with partial resection of the clavicle, though it was difficult to extend the neck due to a previous postoperative neck scar, severe obesity, and short neck. The retropharyngeal mass was then resected transorally.

Transoral videolaryngoscopic surgery was performed according to the procedure reported by Tomifuji et al. [16]. The patient was intubated orotracheally with supine positioning. An FK-WO TORS Laryngo-Pharyngoscope Retractor (Olympus, Tokyo) was positioned so as to provide sufficient working space for RPN dissection. The retractor was then suspended with a holder. A flexible endoscope capable of angulation in 4 directions (Visera LTF-type VP, Olympus) was inserted through the oral cavity while an assistant held and manipulated the endoscope for viewing the surgical field.

To identify RPN, we performed intraoperative ultrasonography with the Aloca Arietta 70 (Hitachi, Tokyo), a flexible laparoscopic transducer manipulated with forceps. We confirmed that the RPN was located in the deep portion of the posterior palatine arch, lateral to the constrictor muscle. The RPN was sharply marginated. Ultrasound showed that the carotid artery was located adjacent to RPN (Fig. 3). Transoral ultrasound helped determine the mobility of RPN relative to the carotid artery, through differential application of pressure to the retropharyngeal mass.

We cut the pharyngeal mucosa and submucosa away from posterior palatine arch and could visualize the constrictor muscle. After severing this muscle, the RPN was visible (Fig. 4). During our procedure, we used ultrasound continually to confirm that the surgical approach to the tumor was appropriate. The transducer is compact in size, and its cable is thin and flexible. Therefore, even in a narrow working space such as the intraoral cavity, we could perform the intraoperative ultrasonography without the need to undock it.

The operator employed a single-use electrosurgical knife with radiofrequency alternating current (KD-600, Olympus) to resect the tumor by transoral videolaryngoscopic surgery. This knife has several characteristics, as follows: a disposable needle, malleable shaft, and adjustable needle length from 2 to $4 \mathrm{~mm}$. We used the Swift mode (output power 30, effect 4) of the high-frequency surgical system (ERVE VIO300D). This procedure could be completed without the need for intraoperative conversion to an open surgery procedure. The transoral videolaryngoscopy surgery lasted $2 \mathrm{~h}$, with little blood loss and no need for blood transfusion, and no tracheostomy was performed either pre- or postoperatively.

No serious hemorrhage or emergent airway compromise was observed during the patient's hospital stay. There were no serious adverse events that required further intervention. The patient began eating food with a paste-like consistency on postoperative day 2 , and resumed a regular diet on postoperative day 5 . The nasogastric tube was removed on postoperative day 2. Postoperative swallowing function was normal with a functional outcome swallowing scale (FOSS) of grade 0. A postoperative videofluoroscopic swallowing study (VFSS) showed no aspiration or penetration. The retropharyngeal tumor in this case was diagnosed pathologically as papillary adenocarcinoma without extranodal spread.

The patient underwent ${ }^{131}$ I therapy postoperatively. She is alive and has remained disease free 20 months after treatment. 
 Oncology}

\section{Discussion}

The retropharyngeal space can be the site of metastatic disease in patients with certain head and neck cancers, especially thyroid cancer and pharyngeal cancer. RPN metastases were diagnosed in $2.24 \%$ patients with thyroid carcinoma [4]. Traditionally, surgical excision of isolated RPN metastases from well-differentiated thyroid carcinoma has been performed via the transcervical and transmandibular approaches [3].

However, these traditional approaches carry the risk of injury to major vascular structures, lower cranial nerves, and the sympathetic chain, as well as potentially causing bone and dental damage. These procedures carry rather high morbidity rates considering the relatively indolent nature of well-differentiated thyroid cancer [7, 17]. Therefore, endoscopic-assisted transoral resection, including transoral robotic surgery for metastatic RPN from well-differentiated thyroid cancer, has recently been described [4-15].

Transoral resection has several advantages, as follows. The operation time may be shorter than with the transcervical approach for an isolated RPN in a previously operated neck [15]. Furthermore, transoral surgery offers more direct access to the tumor, and thus despite adequate retropharyngeal exposure, small tissue injury is less likely compared with transcervical, transparotid, and transmandibular approaches. The patient in this report had advanced-stage thyroid cancer and underwent total thyroidectomy, nerve reconstruction, and neck dissection that included extensive lateral neck lesions, and thus it was expected that if the transcervical approach were used, it would have to be performed through the fibrosis at the previous surgical site. Thus we selected endoscopic transoral surgery to resect the retropharyngeal mass.

However, there are several problems with transoral surgery for metastatic RPN. The retropharyngeal space is a potential space limited by the buccopharyngeal fascia and pharyngeal constrictor muscles anteriorly, and the alar layer of the prevertebral fascia posteriorly, all of which are located medial to the internal carotid artery. Therefore, transoral surgery for metastatic RPN requires resection of the superior constrictor muscle and possible disruption of the upper vagal nerve, glossopharyngeal nerve, and palatal muscle, all of which are important in swallowing function [15]. However, few studies have provided detailed and objective analysis of swallowing function after transoral surgery of retropharyngeal tumors. In this report, VFSS demonstrated no difference in the patient's swallowing function before and after surgery. In addition, this approach carries a potential risk of injury to the internal carotid artery during anatomical localization. Furthermore, it is difficult to localize the tumor.

To solve these problems, we used intraoperative ultrasonography, which may provide several advantages. First, transoral ultrasound clearly revealed the relationship between the mass and the internal carotid artery and internal jugular vein. Transoral ultrasound with power Doppler imaging can also show the internal vascularity associated with the retropharyngeal mass [8]. These advantages enabled the surgeon to confirm the feasibility of transoral surgery and precisely localize the RPN in relation to the adjacent vascular structures, thus improving dissection planning and the accuracy of the incision location. In this patient, transoral ultrasound allowed for the differential application of pressure to the lesion retropharyngeal mass to localize the mass and determine its mobility in relation to the carotid artery. We were able to fully resect the mass without injury of carotid artery. The retropharyngeal space was difficult to fully visualize with external transcervical ultrasound because of the shadowing effect of the mandible. The transoral approach for retropharyngeal tumors is therefore an advantage. 
Intraoperative transoral ultrasound has been reported with the use of an endocavitary probe, finger grip probe, and hockey stick transducer $[18,19]$. However, the finger grip intraoperative transducer could not be moved adequately when applied to the posterior wall, and it was not possible to switch easily from the sagittal to the transverse scan plane [18]. The probes used with the endocavitary probe and hockey stick transducer are too large for the oral cavity, so the surgeon could not see the surgical field or perform manipulation with forceps. We performed ultrasonography with the Aloca Arietta 70 in this patient. This device has a flexible laparoscopic transducer that can be manipulated with forceps (L43K probe). Due to the transducer's small size and thin, flexible cable, we could perform the ultrasonography while resecting the tumor, even in the narrow space of the intraoral cavity, and could confirm precise localization of the RPN in relation to the adjacent vascular structure, thus improving the accuracy of the incision placement.

The finger grip probe and endocavitary probe are both convex-type devices, while the L43K is a linear-type probe. Convex-type probes have a wide and less clear view compared with the linear type. Because transoral surgery takes place in a narrow working space, linear-type ultrasound is ideal.

We effectively performed intraoperative, ultrasound-guided, endoscopic transoral surgery for metastatic RPN from PTC, with complete resection and full preservation of swallowing function.

\section{Acknowledgements}

This work was supported by the Japan Agency for Medical Research and Development (Grant number 15lk0201022h0003).

\section{Statement of Ethics}

This report was approved by the Tottori University institutional review board (160A154). Informed consent was obtained from the patient for the transoral surgery and subsequent publication.

\section{Disclosure Statement}

We have no conflict of interest.

\section{References}

1 Shaha AR: Management of the neck in thyroid cancer. Otolaryngol Clin North Am 1998;31:823-831.

-2 Coskun HH, Ferlito A, Medina JE, Robbins KT, Rodrigo JP, Strojan P, et al: Retropharyngeal lymph node metastases in head and neck malignancies. Head Neck 2011;33:1520-1529.

-3 Otsuki N, Nishikawa T, Iwae S, Saito M, Mohri M, Nibu K: Retropharyngeal node metastasis from papillary thyroid carcinoma. Head Neck 2007;29:508-511.

-4 Shellenberger T, Fornage B, Ginsberg L, Clayman GL: Transoral resection of thyroid cancer metastasis to lateral retropharyngeal nodes. Head Neck 2007;29:258-266.

5 Le TD, Cohen JI: Transoral approach to removal of the retropharyngeal lymph nodes in welldifferentiated thyroid cancer. Laryngoscope 2007;117:1155-1158. 


\section{Case Reports in Oncology}

\begin{tabular}{l|l}
\hline Case Rep Oncol 2017;10:649-655 \\
\hline DOI: $10.1159 / 000478653$ & $\begin{array}{l}\text { C 2017 The Author(s). Published by S. Karger AG, Basel } \\
\text { www.karger.com/cro }\end{array}$ \\
\hline
\end{tabular}

Fujiwara et al.: Ultrasound-Guided Transoral Videolaryngoscopic Surgery for Retropharyngeal Lymph Node Metastasis of Papillary Thyroid Cancer
$>6$

Laccourreye L, Breheret R, Rohmer V, Dubin J, Bizon A: Transoral resection of thyroid cancer metastasis to retropharyngeal lymph node. Ann Otolaryngol Chir Cervicofac 2008;125:309-312.

$>7$ involvement in I-131-negative 18-fluoro-2-deoxyglucose positron emission tomography-positive recurrent thyroid cancer. Skull Base 2009;19:431-436. an guided transoral resection of parapharyngeal retropharyngeal thyroid carcinoma metastases. Head Neck 2011;33:166-170.

$>9$ Buttà L, Lombardi D, Marconi A, Nicolai P: Transoral excision of a retropharyngeal lymph node under rigid endoscopic control. Ann Otol Rhinol Laryngol 2010;119:211-214.

10 Byeon HK, Duvvuri U, Kim WS, Park YM, Hong HJ, Koh YW, et al: Transoral robotic retropharyngeal lymph node dissection with or without lateral oropharyngectomy. J Craniofac Surg 2013;24:11561161.

-11 Moore EJ, Ebrahimi A, Price DL, Olsen KD: Retropharyngeal lymph node dissection in oropharyngeal cancer treated with transoral robotic surgery. Laryngoscope 2013;123:1676-1681.

12 'Malley Jr BW, Quon H, Leonhardt FD, Chalian AA, Weinstein GS: Transoral robotic surgery for parapharyngeal space tumors. ORL J Otorhinolaryngol Relat Spec 2010;72:332-336.

13 Park YM, De Virgilio A, Kim WS, Chung HP, Kim SH: Parapharyngeal space surgery via a transora approach using a robotic surgical system: transoral robotic surgery. J Laparoendosc Adv Surg Tech A 2013;23:231-236.

14 Chan JY, Tsang RK, Eisele DW, Richmon JD: Transoral robotic surgery of the parapharyngeal space: a case series and systematic review. Head Neck 2015;37:293-298.

15 Gaepfert RP, Liu C, Ryan WR: Trans-oral robotic surgery and surgeon-performed trans-oral ultrasound for intraoperative location and excision of an isolated retropharyngeal lymph node metastasis of papillary thyroid carcinoma. Am J Otolaryngol 2015;36:710-714.

-16 Tomifuji M, Araki K, Yamashita T, Shiotani A: Transoral videolaryngoscopic surgery for orophayngeal, hypopharyngeal, and supraglottic cancer. Eur Arch Otorhinolaryngol 2014;271:589-597.

-17 Stojadinovic A, Shoup M, Nissan A, Ghossein RA, Shah JP, Brennan MF, et al: Recurrent differentiated thyroid carcinoma: biological implications of age, method of detection, and site and extent of recurrence. Ann Surg Oncol 2002;9:789-798.

-18 Fornage BD, Edeiken BS, Clayman GL: Use of transoral sonography with an endocavitary transducer in diagnosis, fine-needle aspiration biopsy and intraoperative localization of retropharyngeal masses. AJR Am J Roentgenol 2014;202:481-486.

19 Wong KT, Tsang RK, Tse GM, Yuen EH, Ahuja AT: Biopsy of deep-seated head and neck lesions under intraoral ultrasound guidance. AJNR 2006;27:1654-1657.
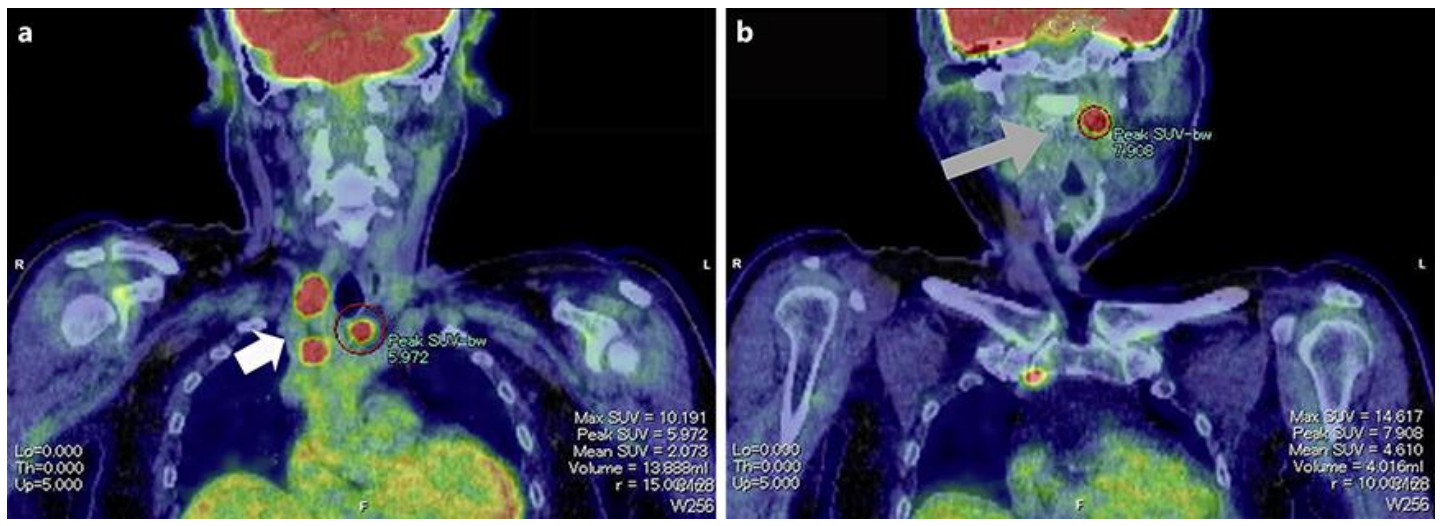

Fig. 1. Positron emission tomography (PET)-computed tomography imaging (coronal section). a Fluorodeoxyglucose (FDG)-PET showed abnormal uptake in the upper mediastinal lymph nodes (white arrow). b FDG-PET showed abnormal uptake in the retropharyngeal lymph node (gray arrow). 


\section{Case Reports in Oncology}

www.karger.com/cro

Fujiwara et al: Ultrasound-Guided Transoral Videolaryngoscopic Surgery for Retropharyngeal Lymph Node Metastasis of Papillary Thyroid Cancer
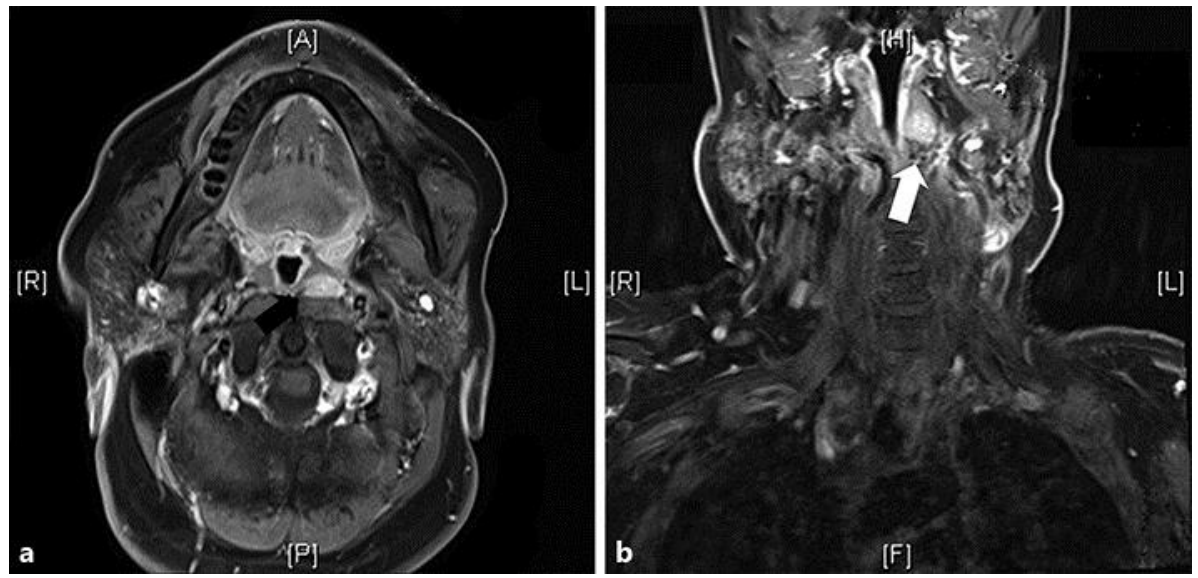

Fig. 2. Magnetic resonance imaging (MRI). Contrast-enhanced T1-weighted MRI of the neck showed a hyperintense mass in the left retropharyngeal space adjacent to internal carotid artery. a Axial section. b Sagittal section.
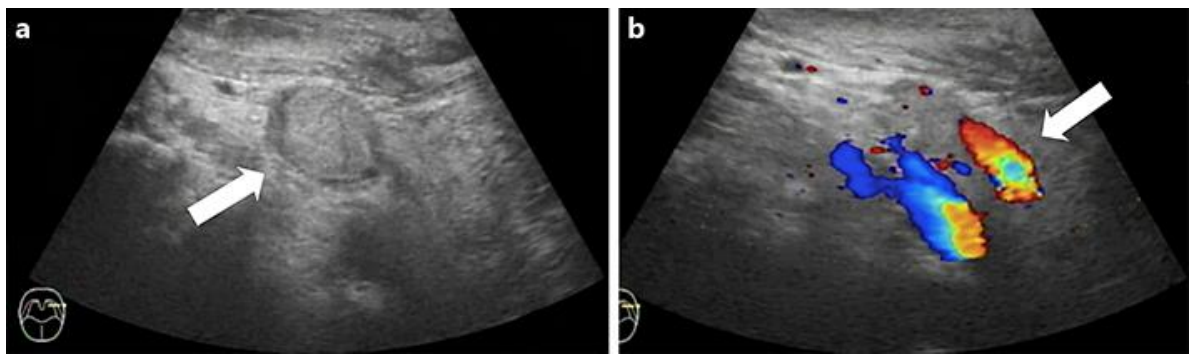

Fig. 3. Transoral ultrasound findings. a Ultrasound showed the retropharyngeal lymph nodes under the constrictor muscle. $\mathbf{b}$ The internal carotid artery was positioned at the deep portion of the retropharyngeal lymph node.
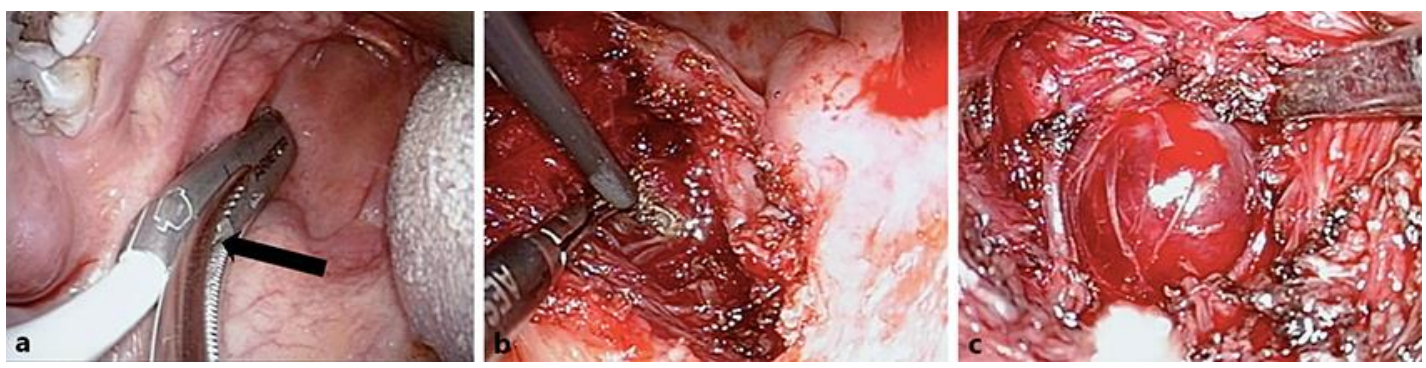

Fig. 4. Intraoperative findings. a The flexible laparoscopic transducer has a handle that can be grasped with forceps. The transducer was used transorally. $\mathbf{b}$ The constrictor muscle was cut using an electrosurgical knife. c The retropharyngeal lymph node was exposed under the constrictor muscle. 